\title{
The assessment of muscle strength symmetry in kayakers and canoeists
}

\author{
Michał Wietrzyński ${ }^{1}$, Joanna Mazur-Różycka², Jan Gajewski ${ }^{1}$, Radosław Michalski², \\ Sebastian Różycki ${ }^{1}$, Krzysztof Buśko ${ }^{3}$
}

${ }^{1}$ Faculty of Physical Education, Józef Piłsudski University of Physical Education, Warsaw, ${ }^{2}$ Institute of Sport, Warsaw, Poland, ${ }^{3}$ Faculty of Physical Education, Health and Tourism, Kazimierz Wielki University, Bydgoszcz, Poland

\section{Summary}

Study aim: To determine and compare the muscle strength profile and muscle strength symmetry of kayakers and canoeists.

Material and methods: A total of 36 male participants participated in the study, including 25 kayakers and 9 canoeists. Measurements of maximum muscle torque were taken under static conditions for 10 muscle groups: flexors and extensors of the elbow, shoulder, knee, hip, and trunk. Muscle torque was allometrically scaled by body mass. To determine the muscle strength profiles of athletes in both disciplines, residual analysis was used. Two methods were utilized to assess and compare the muscle strength symmetry between left and right limbs. The first one is known as intraclass correlation coefficient (ICC). The second one is an asymmetry coefficient proposed by authors.

Results: The study showed that kayakers obtained lower rates of asymmetry indicators than canoeists in most muscle groups. An overall asymmetry coefficient amounted to $0.77 \pm 0.20$ and $0.99 \pm 0.31(p<0.05)$ for kayakers and canoeists, respectively. Moreover, it was observed that the kayakers and canoeists had similar strength profile. The symmetry assessment of maximum muscle torque corresponds to the characteristics of the studied disciplines.

Conclusions: The intraclass correlation coefficient is recommended as a measure of strength symmetry for muscle groups comparisons. The asymmetry coefficient is recommended for comparison of individuals.

\section{Key words: symmetry, maximal muscle torque, men, kayaking, canoeing}

\section{Introduction}

Canoeing and kayaking are 2 sports disciplines that are common subjects in the context of research in the field of anthropometry and somatotype [1], physiology of physical effort [6], and in the evaluation of training effectiveness $[7,8]$. The issue of muscle strength developed by athletes and the impact of the nature of these disciplines on strength distribution in athletes are particularly interesting subjects from a biomechanical point of view. The characteristics of kayaking and canoeing require of athletes special strength preparation [1] that must include the dominant role of the upper limb muscles [1] and the trunk [6] in these sports. For several decades, there have been discussions about methods used to assess muscle strength developed in athletes from different disciplines. In 1968, Fidelus suggested evaluating an athlete's strength by summarizing maximum muscle torque [14]. The concept of relative muscle strength was introduced in order to consider the apparent dependence of maximum muscle torque on body mass. Such an approach seems to be largely justified given that body mass - especially fat free body mass - is the primary determinant of the developed strength. However, many authors have stated $[15,16]$ that a linear relationship between torque and body mass is a simplification of the actual relationship of these variables, which is better reflected in the equation of the power function. This approach is known as allometric scaling.

In kayaking, both upper limbs are used symmetrically in the movement of drawing the paddle while is a seated position; in canoeing, however, a canoeist draws the paddle on one side while kneeling on one leg, which - as is suspected - may imply the presence of strength asymmetry in different muscle groups. Drid et al. [5] indicated that kayakers' muscle strength is distributed symmetrically, but similar results were not reported with regards to canoeists. The attempts to compare the strength symmetry in both disciplines in the same tests were not conducted. Strength training for 
kayakers and canoeists is in some respects quite similar. In both disciplines the greatest focus is placed on building strength in muscle groups of the upper limbs and the trunk. On the other hand, the rowing technique clearly differentiates these two disciplines: kayaking is defined as a symmetrical discipline, while canoeing as an asymmetric discipline. Therefore, an attempt to assess and compare muscle strength asymmetry of kayakers and canoeists was undertaken.

The aim of this study was to determine the profile of muscle strength in kayakers and canoeists, as well as to suggest methods for comprehensively evaluating strength symmetry in these sports disciplines, in particular muscle groups, and in individuals.

\section{Material and methods}

A total of 34 young men, including 25 kayakers and 9 canoeists, participated in the research. Table 1 presents the characteristics of the study participants; no significant differences between the mean values of the analyzed variables were discovered ( $t$ test for independent samples).

The measurements were conducted on a special station that allowed the participant's position to be stabilized and to record the strength of particular muscle groups without the support of muscles not under consideration. The participant pressed the measuring lever for 2-3 seconds using force developed by isometric contraction of the measured muscle group. A special measuring element used at the station, a tensometric torque converter, allowed the results of measurements to be displayed directly in units of torque on the screen of a tensometric amplifier, i.e., in newton meter [N.m]. There was no need to measure the length of the external forces arm (i.e., the distance from the axis of rotation of the lever to the point of application of force by the tested person).

A 10-minute warm-up performed by the test's participants preceded the measurements. The maximum torque values for 10 muscle groups were measured and recorded. Torque for elbow joint flexion and extension was measured in a sitting position. A participant's arm rested on an armrest and was bent $90^{\circ}$ in relation to the body. The value of flexion of a forearm in an elbow joint was $90^{\circ}$. The value of torque while bending and straightening the shoulder joint was measured in a sitting position. A belt placed on a participant's chest provided additional immobilization for a participant's trunk. For shoulder extension and flexion testing, the angle of flexion was, respectively, $70^{\circ}$ and $50^{\circ}$. The measurement of torque values for knee flexion and extension was performed in a sitting position. The measured limb was bent at the knee joint at an angle of $90^{\circ}$. Torque for hip flexion and extension was measured, respectively, while lying on the back and while lying on the stomach. Participants' hip flexor strength was measured while hip and knee flexion was $90^{\circ}$ and participants were holding the edge of the bench while their hands were behind their head. Hip extensor strength was measured using the same values for the angle of the joints; however, participants were lying on their stomach with the edge of bench being at the height of the anterior superior iliac spine, and so flexion in the hip was possible. Torque values for trunk flexors and extensors were measured in a sitting position. A roller placed at the height of the anterior superior iliac spine provided additional stabilization for the trunk. The axis of rotation of the tested joint coincided with the axis of rotation of the torque sensor. Measurements were conducted in the same order for both the right and left sides: first flexion then extension. Participants were asked to develop the greatest possible torque values.

\section{Methods of data analysis}

Statistical analysis of the results was conducted with the STATISTICA statistical software package. To assess muscle strength symmetry in the studied groups, 2 computational methods described later in the paper were used. Strength profiles in both disciplines were calculated by the analysis of residuals, which enabled the expected torque value to be determined for each muscle group. The resulting values were then compared with the values recorded during the measurements. The difference between these two values, called the residual, was the core of further analysis. The expected torque value was calculated by means of allometric scaling of the average value of torque for the left and right sides of the body, taking into account a participant's body mass as well. This approach in calculation is an alternative for determining standards of muscle strength on the basis of relative muscle torque. According to the authors of this paper, the latter approach is a simplification of the real dependence of two variables. The dependence is better

Table 1. General characteristics of the studied group

\begin{tabular}{ccccc}
\hline Dyscypline & Number of subjects & Age [years] & Body mass [kg] & Body weight [cm] \\
\hline Kayaking & 25 & $17.7 \pm 1.0$ & $79.3 \pm 6.6$ & $179.4 \pm 13.9$ \\
Canoe & 9 & $17.1 \pm 1.0$ & $77.9 \pm 9.1$ & $181.1 \pm 6.0$ \\
\hline
\end{tabular}


expressed in the non-linear function of allometric scaling to body mass [8.16]. This function is described by the following formula:

$$
\ln \mathrm{M}_{\mathrm{o}}(\mathrm{m})=\mathrm{a} \ln \mathrm{m}+\mathrm{b},
$$

which after removing the logarithm takes the following form:

$$
\mathrm{M}_{\mathrm{o}}(\mathrm{m})=\mathrm{Bm}^{\mathrm{a}}
$$

where: $\ln$ - natural logarithm, $\mathrm{M}_{\mathrm{o}}$ - expected values of torque, $\mathrm{m}$ - body mass, $\mathrm{a}$ and $\mathrm{b}$ - regression coefficients, $B=e^{b}(e-$ base of natural logarithm $)$.

After calculating the expected value of torque, the residuals were calculated using the following formula:

$$
\mathrm{d}=\ln \mathrm{M}-\ln \mathrm{M}_{\mathrm{o}} .
$$

The standardized residuals were also calculated. Standardization was done to the standard error of regression. Expected torque was calculated separately for each muscle group. This means that each muscle group had a different muscle regression equation (and thus, different regression coefficients). Standardization enabled the residuals for different muscle groups to be compared.

$$
\mathrm{d}^{*}=\frac{\ln \mathrm{M}-\ln \mathrm{M}_{\mathrm{o}}}{\mathrm{SE}}=\frac{\mathrm{d}}{\mathrm{SE}},
$$

where: $\mathrm{d}$ - the residual, $\mathrm{SE}$ - standard error of regression, $\mathrm{n}$ - number of subjects.
To compare the asymmetry of muscle torque of particular muscle groups in kayakers and canoeists, the intraclass correlation coefficient (ICC) calculated for the standardized residuals was used. This coefficient is defined as a measure of compatibility of several independent measurements regardless of their order. In this research, the ICC was calculated while examining the compatibility of measurements of the left and right sides of the body. The value of the coefficient was not influenced by the direction of the asymmetry.

To evaluate the correlation of standardized residuals of particular muscle groups, the Pearson correlation coefficient was calculated. The $t$ test was used to assess the significance of differences between mean values of residuals.

A comprehensive asymmetry assessment in individuals (taking into account all the muscle groups of the limbs) was made on the basis of the suggested asymmetry coefficient:

$$
\varphi=\sqrt{\frac{\sum_{i=1}^{8}\left(d_{L i}^{*}-d_{P i}^{*}\right)^{2}}{8}},
$$

where: $d_{L i}^{*}, d_{P i}^{*}$ - the standardized residuals respective-

\begin{tabular}{|c|c|c|c|c|}
\hline Joint & \multicolumn{2}{|c|}{ Function } & Kayaking $\mathrm{Mm}[\mathrm{Nm}](\mathrm{n}=25)$ & Canoe $\mathrm{Mm}[\mathrm{Nm}](\mathrm{n}=9)$ \\
\hline \multirow{4}{*}{ Elbow } & \multirow{2}{*}{$\mathrm{L}$} & $\mathrm{F}$ & $89.0 \pm 12.0$ & $77.9 \pm 13.2$ \\
\hline & & $\mathrm{E}$ & $68 \pm 12.9$ & $61.8 \pm 9.8$ \\
\hline & \multirow{2}{*}{$\mathrm{R}$} & $\mathrm{F}$ & $85.8 \pm 12.1$ & $87.0 \pm 16.0$ \\
\hline & & $\mathrm{E}$ & $64 \pm 14.3$ & $66.4 \pm 11.5$ \\
\hline \multirow{4}{*}{ Shoulder } & \multirow{2}{*}{$\mathrm{L}$} & $\mathrm{F}$ & $72.6 \pm 10.9$ & $73.4 \pm 11.8$ \\
\hline & & $\mathrm{E}$ & $99.8 \pm 15$ & $96.3 \pm 8.6$ \\
\hline & \multirow{2}{*}{$\mathrm{R}$} & $\mathrm{F}$ & $79.2 \pm 14.7$ & $81.6 \pm 10$ \\
\hline & & $\mathrm{E}$ & $98.3 \pm 14.8$ & $102 \pm 15.2$ \\
\hline \multirow{4}{*}{ Knee } & \multirow{2}{*}{$\mathrm{L}$} & $\mathrm{F}$ & $122 \pm 19.3$ & $102 \pm 15.6$ \\
\hline & & $\mathrm{E}$ & $290 \pm 94$ & $300 \pm 60$ \\
\hline & \multirow{2}{*}{$\mathrm{R}$} & $\mathrm{F}$ & $131 \pm 21.7$ & $116 \pm 25.5$ \\
\hline & & $\mathrm{E}$ & $295 \pm 93.1$ & $318 \pm 63.4$ \\
\hline \multirow{4}{*}{ Hip } & \multirow{2}{*}{$\mathrm{L}$} & $\mathrm{F}$ & $137 \pm 23.8$ & $128 \pm 27.4$ \\
\hline & & $\mathrm{E}$ & $535 \pm 113$ & $513 \pm 101$ \\
\hline & \multirow{2}{*}{$\mathrm{R}$} & $\mathrm{F}$ & $138 \pm 20.6$ & $130 \pm 23.7$ \\
\hline & & $\mathrm{E}$ & $544 \pm 115$ & $512 \pm 92$ \\
\hline \multirow{2}{*}{ Trunk } & & $\mathrm{F}$ & $241 \pm 30$ & $223 \pm 44$ \\
\hline & & $\mathrm{E}$ & $666 \pm 156$ & $717 \pm 146$ \\
\hline
\end{tabular}
ly for the left and right side for the $i$-th $(i=1,2, \ldots 8)$ muscle groups.

Table 2. Mean $( \pm \mathrm{SD})$ values of maximum torque $(\mathrm{Mm}[\mathrm{Nm}])$ for flexor muscles $(\mathrm{F})$ and extensor muscles $(\mathrm{E})$ of the limbs (right $-\mathrm{R}$; left $-\mathrm{L})$ in particular joints in the group of kayakers $(\mathrm{n}=35)$ and canoeists $(\mathrm{n}=9)$ 


\section{Results}

The mean values of maximum muscle torque $( \pm \mathrm{SD})$ for the right and the left limb are presented in Table 2.

Mean values of residuals of maximum muscle torque $( \pm \mathrm{SD})$ are shown in Table 3 . The only statistically significant difference in the mean values of residuals between the studied groups was found in the torque of the knee joint extensor.
The profiles of muscle strength in individuals practicing kayaking and canoeing are presented in Figure 1. They have been determined on the basis of standardized residuals that were calculated for each muscle group.

The following symbols were assumed: elbow flexion/extension (EF/EE); shoulder flexion/extension (SF/SE); knee flexion/extension(KF/KE); hip flexion/ extension (HF/HE); trunk flexion/extension (TF/TE).

Figure 2 presents values of the intraclass correlation coefficients (ICCs) for standardized residuals of muscle

Table 3. The mean values $( \pm \mathrm{SD}$ ) of residuals of maximum muscle torque logarithms for the muscle groups on the left and right sides of the body that perform flexion movement (F) and extension movement (E) in the elbow, shoulder, knee and hip joints, as well as the trunk, in kayakers and canoeists

\begin{tabular}{cccccc}
\hline \multirow{2}{*}{ Joint } & \multirow{2}{*}{ Function } & \multicolumn{2}{c}{ Kayaking $(\mathrm{n}=25)$} & \multicolumn{2}{c}{ Canoe $(\mathrm{n}=9)$} \\
& & $\mathrm{Mm}[\mathrm{Nm}]$ & Residuals [-] & $\mathrm{Mm}[\mathrm{Nm}]$ & Residuals [-] \\
\hline \multirow{2}{*}{ Elbow } & $\mathrm{F}$ & $88 \pm 12$ & $0.012 \pm 0.119$ & $82.4 \pm 14.9$ & $-0.033 \pm 0.081$ \\
& $\mathrm{E}$ & $66 \pm 13.6$ & $0.001 \pm 0.174$ & $64.1 \pm 10.7$ & $-0.001 \pm 0.113$ \\
Shoulder & $\mathrm{F}$ & $76 \pm 13.2$ & $-0.011 \pm 0.122$ & $77.5 \pm 11.4$ & $0.032 \pm 0.130$ \\
& $\mathrm{E}$ & $99 \pm 14.8$ & $-0.006 \pm 0.120$ & $99.3 \pm 12.4$ & $0.017 \pm 0.050$ \\
Knee & $\mathrm{F}$ & $126 \pm 20.9$ & $0.035 \pm 0.120$ & $109 \pm 21.8$ & $-0.098 \pm 0.0155$ \\
& $\mathrm{E}$ & $293 \pm 73$ & $-0.024 \pm 0.248^{*}$ & $309 \pm 60.6$ & $0.067 \pm 0.140$ \\
Hip & $\mathrm{F}$ & $138 \pm 22$ & $0.012 \pm 0.154$ & $129 \pm 24.9$ & $-0.035 \pm 0.093$ \\
& $\mathrm{E}$ & $540 \pm 115$ & $0.005 \pm 0.181$ & $512 \pm 93.5$ & $-0.015 \pm 0.126$ \\
Trunk & $\mathrm{F}$ & $241 \pm 29.7$ & $0.018 \pm 0.130$ & $223 \pm 43.7$ & $-0.050 \pm 0.132$ \\
& $\mathrm{E}$ & $666 \pm 156$ & $-0.029 \pm 0.173$ & $717 \pm 146$ & $0.080 \pm 0.102$ \\
\hline
\end{tabular}

* - significantly different than in the canoeists $(p<0.05)$

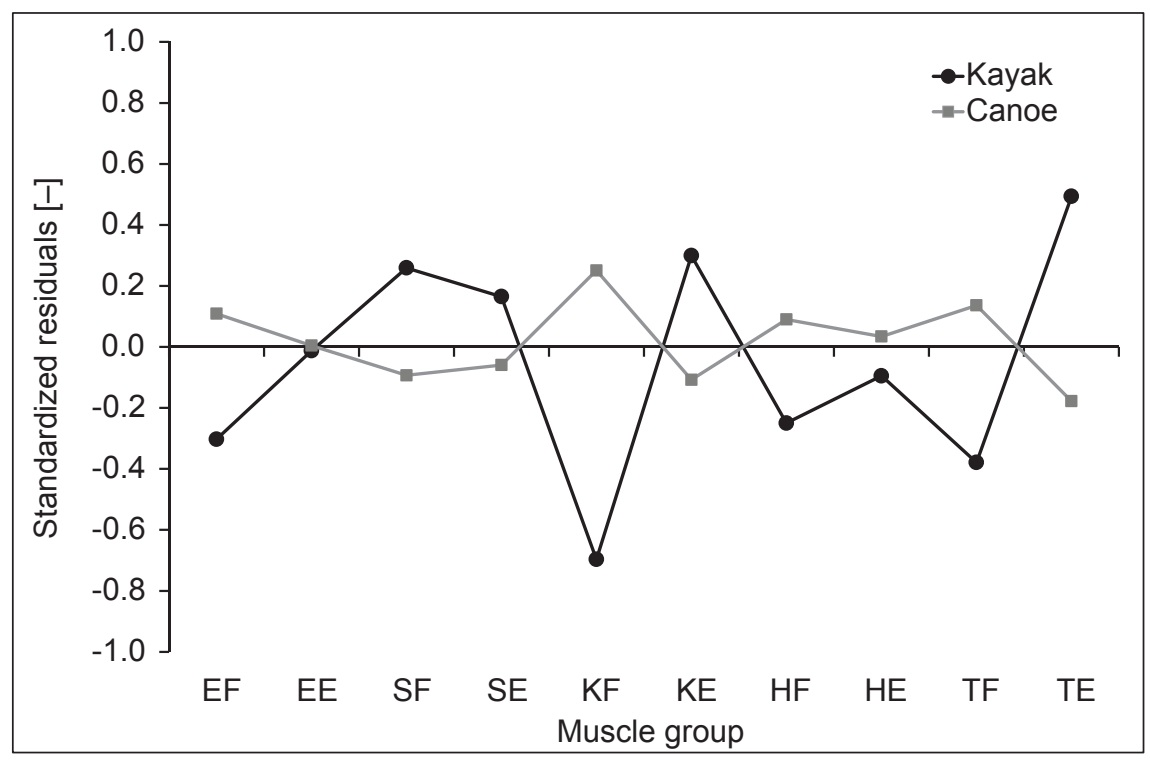

Fig. 1. Mean strength profiles of men practicing kayaking and canoeing. The asterisk indicates the statistically significant differences observed at the significance level $\alpha=0.05$ 


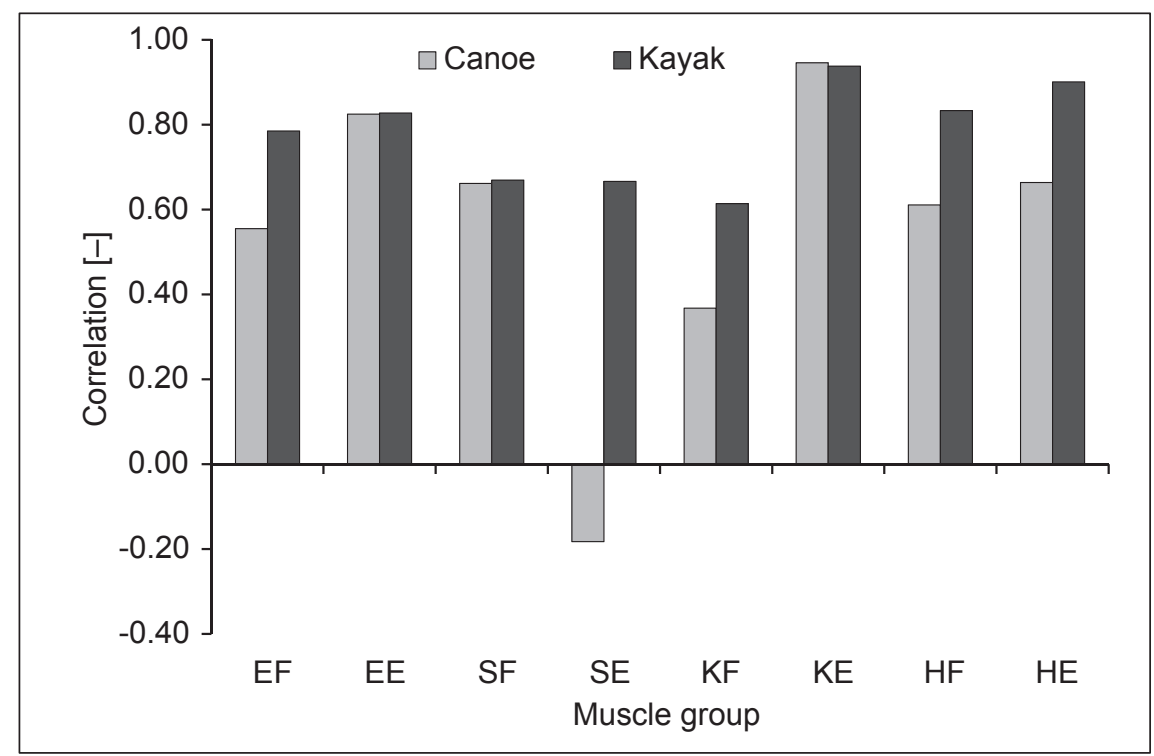

Fig. 2. Intraclass correlation coefficients (ICCs) of standardized residuals of maximal joint torques for left and right body side in the kayakers and canoeists

torque for the left and right sides of the body in kayakers and canoeists. The higher the value of the ICC, the greater the symmetry of maximum torque of a particular muscle group in the studied discipline. The group of knee joint flexor muscles demonstrated high levels of symmetry in both disciplines. The lowest results were observed in the shoulder extensor muscles in canoeists and the group of knee flexor muscles in kayakers.

The mean value of the overall asymmetry coefficient $\varphi$ (formula 5) for canoeists was $0.99 \pm 0.31$; for kayakers, $0.77 \pm 0.20$. The mean difference was significant $(p<0.05)$. According to this assessment, higher asymmetry was discovered in the group of canoeists.

\section{Discussion}

Measuring the maximum torque of muscles in static conditions is a standard procedure for a study of muscle strength due to the reliability of the obtained results and the simplicity of the research mechanism. The movements most frequently used for measurements are flexion and extension in the major joints in the body: elbow, shoulder, knee, hip, as well as the flexion and extension of the trunk. Many authors use the above procedure in an analysis of muscle strength in both professional athletes as well as in recreational practitioners of sport $[4,3,12]$.

This research determined the muscle strength profile understood as the mutual relationship of the maximum torque of particular muscle groups [2]. The muscle strength profile was also referred to as the topography of strength [11] and the topography of muscle torque [3]. A comparison of muscle strength profiles in kayakers and canoeists is presented in Figure 1. It was assumed that the profiles in both disciplines differ due to different body positions maintained in kayaks and canoes (kayakers sit with their back straight up, canoeists kneel on one leg) as well as the asymmetrical work involved during paddling (kayakers with a double-bladed paddle, canoeists with an one-bladed paddle). However, the strength profile of the studied groups did not differ significantly. The lack of significant differences might stem from the fact that in both disciplines strength training of the upper limbs and trunk plays a very important role $[1,6]$. This includes the development of the same motor features by using similar methods during practice sessions. It seems, therefore, that the position of a player in a kayak doesn't significantly affect the distribution of strength in different muscle groups, with the exception of the knee flexors. The differences between the disciplines with regards to the knee flexors are probably due to different positioning of the lower limbs in a kayak versus a canoe while paddling. The differences also might be related to the relatively minor attention attached to building up the strength of these muscles during training.

The paper proposes a method for assessing the strength symmetry of kayakers and canoeists using the intraclass correlation coefficient (ICC). According to the authors, analyzing this type of correlation is an interesting alternative to the traditional indicators used in analyzing asymmetry $[12,13]$. Figure 2 presents a comparison of the results for the kayakers and canoeists. Studies have shown that kayakers have a more 
balanced distribution of strength that is characteristic for muscle work performed while paddling. One of the elements that affects the symmetry of strength is the training method used in kayaking. The greatest importance is placed on developing the trunk and upper limbs muscles [9]. Canoeists and kayakers are characterized by similar values of the intraclass correlation coefficient for extension at the elbow joint, for flexion at the shoulder joint, and extension at the knee. However, particularly low values of this coefficient that assesses symmetry were described in canoeists for the extension at the shoulder and flexion at the knee. It can be assumed that canoeists' paddling technique (one-bladed paddle) and a kneeling position with one leg, determine the unilateral work and directly cause the observed asymmetry. To summarize, kayakers are characterized by high strength symmetry in all muscle groups, while the distribution of strength in canoeists is distinctively asymmetrical.

The research also determined the asymmetry coefficient that was calculated with the aforementioned formula (5). This is a general indicator calculated, separately for kayakers and for canoeists, on the basis of standardized residuals for all muscle groups. The authors of the paper have proposed this method as a way to comprehensively assess strength asymmetry in individual participants of research. The reasoning for the procedure is founded on the characteristics of the two studied disciplines, which indicate the possibility of developing muscle strength asymmetry. Drid et al. [5] undertook the issue of asymmetry by studying judokas, wrestlers, and people who were not training. To assess muscle strength asymmetry, maximum torque of particular muscle groups was compared. In the paper, all muscle groups of the upper and lower limbs were included while assessing asymmetry.

All the aforementioned calculation methods were used with kayakers and canoeists. The characteristics of these disciplines indicated great muscle strength as well as symmetry (in the case of kayakers), and asymmetry (in the case of canoeists) of muscle strength distribution. The results confirmed this assumption and indicated the accuracy and reliability of the proposed coefficients (muscle strength symmetry of particular muscle groups in the studied group of people - intraclass correlation coefficient (ICC); the comprehensive assessment of strength asymmetry of individuals - formula No. 5). The methods used in this research to compare the strength asymmetry in participants of different sports disciplines established the prospects for future further studies.

To summarize: kayakers and canoeists presented similar profiles of strength (strength distribution of different muscle groups). It was concluded that the muscle strength distribution in canoeists is significantly more asymmetric than in kayakers. To assess the strength symmetry of particular muscle groups understood as a characteristic feature of the group, the paper suggested utilizing the intraclass correlation coefficient (ICC) to .

\section{References}

1. Akca F., Muniroglu S. (2008) Anthropometric - somatotype and strength profiles and on-water performance in Turkish elite kayakers. Int. J. Appl. Sports Sci., 1: 22-34.

2. Bober T., Pietraszewski B. (1996) Strength of muscle groups in swimmers. Biol. Sport, 13: 155-164.

3. Buśko K., Nowak A. (2008) Changes of maximal muscle torque and maximal power output of lower extremities in male judoists during training. Hum. Movement, 9: 111-115.

4. Buśko K., Gajewski J. (2011) Muscle strength and power of elite female and male swimmers. Baltic Journal of Health and Physical Activity, 3: 13-18.

5. Drid P., Drapsin M., Trivic T., Lukač D., Obadov S., Milosevic Z. (2009) Asymmetry of muscle strength in elite athletes. Biomed. Human Kinetics, 1: 3-5.

6. Fekete M., Coach H. (1998) Periodized strength training for sprint kayaking/canoeing. Strength Cond., 20: 8-14.

7. Fidelus K., Skorupski L. (1970) Wielkość momentów sił mięśniowych w poszczególnych stawach u zawodników różnych dyscyplin sportowych. Sympozjum Teorii Techniki Sportowej. Warszawa 26-28.11.1968, 128-140.

8. Gajewski J., Buśko K., Mazur J., Michalski R. (2011) Application of allometry for determination of strength profile in young female athletes from different sports. $B i$ ology of Sport, 28: 239-243.

9. Garcia-Pallares J., Carrasco L., Diaz A., SanchezMediana L. (2009) Post season detraining effects on physiological and performance parameters in top-level kayakers: comparison of two recovery strategies. J. Sports Sci. Med., 8: 622-628.

10. Garcia-Pallares J., Garcia-Fernandez M., SanchezMedina L., Izquierdo M. (2010) Performance changes in world-class kayakers following two different training periodization models. Eur. J. Appl. Physiol., 110: 99-107.

11. Jaric S., Mirkov D., Markovic G. (2005) Normalizing physical performance tests for body size: a proposal for standardization. J. Strength Cond. Res., 19: 467-474

12. McKean M.R., Burkett B. (2010) The relationship between joint range of motion, muscular strength, and race time for sub-elite flat water kayakers. J. Sci. Med. Sport, 13: 537-542.

13. Perry M.C., Carville S.F., Smith H., Chirstopher I., Rutherford O.M., Newham Di J. (2007) Strength, power 
output and symmetry of leg muscles: effect of age and history of falling. Eur. J. Appl. Physiol., 100: 553-561.

14. Trzaskoma Z, Trzaskoma Ł. (2006) Structure of maximal muscle strength of lower extremities in highly experienced athletes. Phys. Edu. Sport, 50: 73-78.

15. Trzaskoma Z, Trzaskoma Ł. (2001) The proportion between maximal torque of core muscles in male and female athletes. Acta Bioeng. Biomech., 3(Suppl. 2): 601-606.
16. Vanderburgh P.M., Mahar M.T., Chou C.H. (1995) Allometric scaling of grip strength by body mass in college-age men and women. Res. Q. Exerc. Sport, 66: 80-84.

Received 02.07.2012

Accepted 03.09.2013

(C) University of Physical Education, Warsaw, Poland 